\title{
LspA inactivation in Mycobacterium tuberculosis results in attenuation without affecting phagosome maturation arrest
}

\begin{abstract}
Correspondence
Peter Sander

psander@immv.uzh.ch
\end{abstract}

Received 28 March 2008

Revised 19 June 2008

Accepted 27 June 2008

\author{
Silvana K. Rampini, ${ }^{1}$ Petra Selchow, ${ }^{1}$ Christine Keller, ${ }^{2}$ Stefan Ehlers, ${ }^{2}$ \\ Erik C. Böttger ${ }^{1,3}$ and Peter Sander ${ }^{1,3}$ \\ ${ }^{1}$ Institut für Medizinische Mikrobiologie, Universität Zürich, Gloriastrasse 32, CH-8006 Zurich, \\ Switzerland \\ ${ }^{2}$ Molecular Infection Biology, Research Center Borstel, D-23845 Borstel, Germany
${ }^{3}$ Nationales Zentrum für Mykobakterien, Gloriastrasse 30, CH-8006 Zurich, Switzerland
}

The success of Mycobacterium tuberculosis depends on its ability to survive within host macrophages. Here, M. tuberculosis avoids the acidic, hydrolytically competent environment of the phagolysosome by arresting phagosome maturation. Having shown previously that a $M$. tuberculosis mutant deficient in lipoprotein signal peptidase (LspA) is strongly attenuated in vivo in a mouse model of infection, we now studied putative mechanisms involved in attenuation of the IspA : : aph mutant at a cellular level. In this work we investigated the ability of the mutant to interfere with two host defence mechanisms, i.e. Toll-like receptor (TLR)2-dependent immune response and phagosome maturation. While mycobacterial lipoproteins have been reported to trigger a TLR2 signalling pathway critical for innate immune responses, we found that growth control of the IspA : : aph mutant was independent of TLR2. In addition, the IspA : : aph mutant arrested phagosome maturation to an extent similar to that of the wild-type, as measured by lysosomal-associated membrane protein 1 (LAMP1) co-localization and intraphagosomal pH. These observations demonstrate severe attenuation even in the presence of arrested phagosome maturation, and point to a role for the early phagosome in growth restriction of the M. tuberculosis IspA mutant.

\section{INTRODUCTION}

Mycobacterium tuberculosis is a major threat to human health, accounting for 1.6 million deaths and 8.8 million new infections in 2005 (World Health Organization fact sheet; http://www.who.int/mediacentre/factsheets/fs104/ en/). The bacteria show a remarkable ability to survive and replicate within macrophages. A multitude of virulence factors contribute to the intracellular survival of pathogenic mycobacteria, such as the physical barrier of the mycobacterial cell wall and the ability of the bacilli to avoid activation of $\mathrm{T}$ cells by manipulation of the host immune

Abbreviations: BMDM, bone marrow-derived macrophages; IFN, interferon; LAMP1, lysosomal-associated membrane protein 1; PIM, phosphatidylinositol mannoside; TLR, Toll-like receptor; TNF- $\alpha$, tumour necrosis factor- $\alpha$.

Four supplementary figures showing kinetics of in vivo growth of $M$. tuberculosis wild-type and mutants in spleen over a 108 day period, restoration of wild-type growth in IFN- $\gamma$-activated BMDM by complementation of $M$. tuberculosis IspA, growth of wild-type, mutant and complemented strains in Middlebrook 7H9-ADC, and TNF- $\alpha$ secretion by BMDM in response to infection with wild type and mutant strains, are available with the online version of this paper. response (reviewed by Chua et al., 2004; Russell, 2001). Among the most striking and prominent features of $M$. tuberculosis is its ability to arrest phagosome maturation, creating an intracellular niche with characteristics of early endosomes suitable for replication of the bacteria (Huynh \& Grinstein, 2007; Russell, 2001; Vergne et al., 2004).

Lipoproteins are a class of secreted, lipidated proteins ubiquitously present in bacteria. Lipoproteins are involved in a variety of functions including cell wall synthesis, nutrient uptake, sensing and transmembrane signalling, and adhesion (Sutcliffe \& Russell, 1995). Lipidation is thought to allow anchoring of these proteins to the cell surface. Lipoproteins are characterized by the presence of a consensus sequence that directs post-translational modifications. One step of the post-translational modifications depends on the lipoprotein signal peptidase (LspA), which cleaves off the signal peptide from immature prolipoproteins (Dev \& Ray, 1984; Sander et al., 2004; Sankaran \& $\mathrm{Wu}, 1994)$. Mycobacterial lipoproteins have been found in both the plasma membrane and the cell wall fraction, with their correct localization depending on LspA (Mawuenyega et al., 2005; Rezwan et al., 2007a, b). A M. tuberculosis 
mutant deficient in LspA ( $\operatorname{spA}:: a p h)$ shows a very severe reduction in c.f.u. in lungs and complete absence of lung pathology in a mouse infection model (Sander et al., 2004). The mechanisms that underlie attenuation remain to be elucidated; however, the phenotype of the lspA::aph mutant suggests strong interactions of lipoproteins with prominent host defence mechanisms.

Mycobacterial lipoproteins are recognized by the Toll-like receptor (TLR)2 (Brightbill et al., 1999), which belongs to the family of pattern recognition receptors (Rock et al., 1998). TLR2 is the core subunit of heterodimeric receptors that recognize lipidated cellular components: TLR2/1 binds tri-acylated mature lipoproteins, while TLR2/6 recognizes di-acyl moieties (Jin et al., 2007). Pathogen recognition through TLR in principle serves three functions: sensing the presence and type of the pathogen, provoking an immediate anti-pathogen response and stimulating the development of a long-lasting adaptive response (Kanzler et al., 2007). The response to TLR signalling is determined by factors specific to individual cell types and the nature of the ligand. It can include cell differentiation, proliferation or apoptosis, and secretion of cytokines, prominently interferons (IFNs) and tumour necrosis factor (TNF)- $\alpha$ (Kanzler et al., 2007). TLR2 contributes to innate resistance against M. tuberculosis (Reiling et al., 2002). In vitro investigations suggest that the triggering of TLR2 by $M$. tuberculosis may represent a double-edged sword: shortterm signalling through TLR2 activates macrophages and initiates inflammation via TNF- $\alpha$ secretion that may help to control the acute infection (Underhill et al., 1999); prolonged TLR2 signalling in macrophages results in downregulation of major histocompatibility complex (MHC)-II antigen processing, suppressing an acquired immune response (Gehring et al., 2004; Noss et al., 2001). The link between TLR2 triggering and phagosome maturation is controversial: Blander \& Medzhitov (2004) reported that TLR stimulation promotes phagosome maturation, while Yates \& Russell (2005) provided evidence that TLR2 or TLR4 stimulation does not affect phagosome acidification or phago-lysosomal fusion.

The phagocytic machinery of macrophages, i.e. phagocytosis and phagosome maturation, is a complex defence mechanism of the innate immune system. Following internalization, phagosomes mature to phago-lysosomes. The maturation process depends on vesicle fusion and fission. It involves acquisition, loss and modification of defined host proteins, and ultimately results in acidification of the phagosomal lumen to $\mathrm{pH}<5$ and generates an environment in which microbes are exposed to lytic enzymes and reactive oxygen and nitrogen intermediates (Huynh \& Grinstein, 2007; Schnappinger et al., 2003; Vieira et al., 2002). Pathogenic mycobacteria interfere with the phagosome maturation process (Armstrong \& Hart, 1971; Clemens \& Horwitz, 1995; Hasan et al., 1997; Via et al., 1997; Xu et al., 1994). The mycobacterial phagosome retains the early endosomal GTPase rab5 (Clemens et al., 2000; Kelley \& Schorey, 2003; Via et al., 1997) and access to transferrin within the rapid recycling pathway (Clemens \& Horwitz, 1996; Sturgill-Koszycki et al., 1996). It does not acquire early endosomal antigen 1 (Fratti et al., 2001), late endosomal/lysosomal markers [such as rab7, lysosomalassociated membrane protein 1 (LAMP1) and CD63] (Chua et al., 2004; Russell, 2001) or vacuolar proton ATPase (Sturgill-Koszycki et al., 1994), and it only acidifies mildly to about pH 6.4 (Oh \& Straubinger, 1996; Russell et al., 2005; Sturgill-Koszycki et al., 1994). Although providing only few carbohydrates and little iron, the maintenance of $M$. tuberculosis in a compartment with early endosomal characteristics is thought to provide a favourable environment suitable for intracellular replication (Huynh \& Grinstein, 2007; Russell, 2001; Schnappinger et al., 2003; Vergne et al., 2004). Upon activation with IFN- $\gamma$, macrophages restrict growth of intracellular mycobacteria; growth restriction is associated with phagosome maturation and further acidification (Hostetter et al., 2002; MacMicking et al., 2003; Schaible et al., 1998; Via et al., 1998). Phagosome maturation arrest is specific to live mycobacteria. Heat-killed mycobacteria undergo phagosome maturation and the intraphagosomal $\mathrm{pH}$ stabilizes at about 5.8 (Russell et al., 2005). The mechanisms involved in phagosome maturation arrest are still elusive. Phagosome maturation arrest is in part dependent on lipid components of the mycobacterial cell wall, such as lipoarabinomannan, phosphatidylinositol mannosides (PIMs) and cord factor (Anes et al., 2003; Fratti et al., 2001, 2003; Indrigo et al., 2003; Vergne et al., 2003, 2004). Additional factors involved are the acid phosphatase SapM (Saleh \& Belisle, 2000; Vergne et al., 2005), the bacterial serine/threonine kinase PknG (Walburger et al., 2004), and the zinc-metalloprotease Zmp1 (Master et al., 2008).

The strong attenuation of the M. tuberculosis $\operatorname{lsp} A:: a p h$ mutant (Sander et al., 2004) prompted us to investigate the mechanism(s) involved in loss of virulence. We here investigated the ability of the mutant to interfere with two innate host defence mechanisms, i.e. TLR2 signalling and phagosome maturation.

\section{METHODS}

Bacterial strains and growth conditions. $M$. tuberculosis $\mathrm{H} 37 \mathrm{Rv}$ \#1424, a derivative of $M$. tuberculosis H37Rv, was used as wild-type strain in this study. The isogenic lspA::aph knockout mutant and lspA::aph-lspA complemented mutant have been described previously (Sander et al., 2004). Strains were grown at $37{ }^{\circ} \mathrm{C}$ on Middlebrook 7H10 agar (Difco) supplemented with OADC (0.05\% oleic acid, $5 \%$ BSA fraction V, $2 \%$ glucose, $0.004 \%$ beef catalase, $0.85 \% \mathrm{NaCl}$; Difco) or in settling cultures at $37{ }^{\circ} \mathrm{C}$ in Middlebrook 7H9 broth (Difco) supplemented with OADC and $0.05 \%$ Tween 80; antibiotics were added when appropriate $\left(50 \mu \mathrm{g} \mathrm{kanamycin} \mathrm{ml}^{-1}\right.$, $25 \mu \mathrm{g}$ hygromycin $\mathrm{ml}^{-1}$ ). For growth kinetics, strains at midexponential phase in roller bottles without antibiotics were diluted to $\mathrm{OD}_{600} 0.01$ in Middlebrook 7H9 supplemented with ADC [5\% albumin, $2 \%$ dextrose, $0.0003 \%$ catalase (beef)] and $0.05 \%$ Tween 80 , and $\mathrm{OD}_{600}$ was monitored over 10-12 days. For infection experiments, strains were grown in $7 \mathrm{H} 9$ broth supplemented with 
OADC and $0.05 \%$ Tween 80 to mid-exponential phase in roller bottles without antibiotics. Heat-inactivated M. tuberculosis was used as a control. Heat-inactivation was performed at $85{ }^{\circ} \mathrm{C}$ for $30 \mathrm{~min}$; inactivation was confirmed by plating on $7 \mathrm{H} 10$. All procedures were done under biosafety level III conditions.

Cells and growth conditions. Macrophages were derived from bone marrow of over eight-week-old specific-pathogen-free female BALB/c or C57BL/6 mice (both from Harlan) maintained in individual ventilated cages at Biologisches Zentrallabor (BZL), University of Zurich. Animal procedures were approved by the Kantonales Veterinäramt (Zurich). Bone marrow stem cells were differentiated at $37{ }^{\circ} \mathrm{C}$ in $5 \% \mathrm{CO}_{2}$ for 7 days in Dulbecco's modified Eagle's medium (DMEM) supplemented with $10 \%$ fetal calf serum (FCS) (Brunschwig), $10 \%$ L-cell conditioned medium and $1 \%$ penicillin/ streptomycin $\left(10000 \mathrm{U} \mathrm{ml}^{-1} / 10000 \mu \mathrm{g} \mathrm{ml}^{-1}\right.$ ) (Gibco) on Petri dishes (Greiner). Viability controls using trypan blue exclusion revealed over $90 \%$ viable cells. During infection assays, cells were maintained in DMEM supplemented with $10 \%$ FCS, a medium which does not support mycobacterial multiplication (Bryk et al., 2008). The mouse monocyte-macrophage cell line J774A.1 was also maintained in DMEM supplemented with $10 \%$ FCS.

Mouse infection experiments. Female 6-8-week-old specificpathogen-free C57BL/6 wild-type or TLR2 ${ }^{-1-}$ mice back-crossed at least 10 times to a C57BL/6 background were maintained under biosafety level III conditions in individually ventilated cages. For infection experiments, $M$. tuberculosis cultures grown to midexponential phase were harvested, aliquoted and frozen at $-80{ }^{\circ} \mathrm{C}$. After thawing, viable cell counts were determined by plating serial dilutions on Middlebrook 7H10 agar supplemented with OADC. To ensure proper dispersion of $M$. tuberculosis, the thawed bacterial suspension was diluted appropriately and drawn through a nonpyrogenic needle (Microlance 3, BD) prior to every infection experiment. Mice were aerogenically infected by producing an aerosol of $5.5 \mathrm{ml}$ of a solution containing $10^{6}$ c.f.u. $\mathrm{ml}^{-1}$ M. tuberculosis in an aerosol generation device (Glas-Col) for $30 \mathrm{~min}$, calibrated to seed $\sim 1000$ c.f.u. of the respective M. tuberculosis strain. The implanted inocula of the wild-type, mutant and complemented strains were confirmed by determination of c.f.u. in the lungs 1 day after infection. At each time point of analysis, lungs from five sacrificed animals were removed, weighed and homogenized. Serial dilutions of organ homogenates were plated on 7H10 agar supplemented with OADC, and c.f.u. were calculated as the arithmetical mean of duplicate determinations and geometric means of the groups at the indicated time points. All experiments performed were in accordance with the German Animal Protection Law and were approved by the Animal Research Ethics Board of the Ministry of Environment, Nature Protection and Agriculture (Kiel) and an institutional review board.

Intracellular growth experiments. Fully differentiated bone marrow-derived macrophages (BMDM) were seeded in 24-well plates (Nunc) at $1 \times 10^{5}$ cells per well and maintained in DMEM supplemented with $10 \%$ FCS for 72 h. For experiments with activated macrophages, $100 \mathrm{U} \mathrm{ml}^{-1}$ recombinant murine IFN- $\gamma$ (Lucerna Chem) was added to the medium when cells were seeded, and the IFN- $\gamma$ level was maintained throughout the chase period. Bacterial suspensions were prepared using cultures grown in roller bottles without antibiotics. Macrophages were infected at $37{ }^{\circ} \mathrm{C}$ in $5 \% \mathrm{CO}_{2}$ for $2 \mathrm{~h}$ at an m.o.i. of 0.5 bacteria per cell in triplicate. Extracellular bacteria were removed by washing each well three times with DMEM. The number of viable intracellular bacteria was determined at day 0 , $1,3,5,7$ and 9 after infection by lysing adherent cells with ice-cold water and $0.04 \%$ SDS. Lysates were plated in serial dilutions on $7 \mathrm{H} 10$ OADC and c.f.u. were counted after 3 weeks of incubation at $37{ }^{\circ} \mathrm{C}$. Plating of the culture medium in preceding experiments had excluded significant extracellular bacterial growth (data not shown).
Measurement of TNF- $\alpha$ production. Supernatants from control and infected BMDM of BALB/c mice were harvested at 5, 24, 48 and $72 \mathrm{~h}$ post-infection, centrifuged at 4000 r.p.m. and aliquots were stored at $-70{ }^{\circ} \mathrm{C}$. Quantification of c.f.u. confirmed that the numbers of bacteria in the inocula were similar for the two strains (data not shown). TNF- $\alpha$ was detected by ELISA (Quantikine, R\&D Systems) according to the manufacturer's instructions. Recombinant TNF- $\alpha$ (R\&D Systems) was used to generate standard curves. Mock infection with culture medium served as a negative control. Each condition was measured in duplicate and in four independent experiments from distinct cell preparations.

Intracellular co-localization experiments. BMDM of BALB/c mice were cultured on $0.7 \mathrm{~mm}$ glass coverslips (Assistent) in 24-well plates at $1.5-2 \times 10^{5}$ cells per well with or without $100 \mathrm{U} \mathrm{ml}^{-1}$ recombinant murine IFN $-\gamma$. Washed mycobacteria were labelled with FITC (SigmaAldrich) by incubation at $37^{\circ} \mathrm{C}$ for $30 \mathrm{~min}$ in PBS, pH 7.4, containing $10 \mathrm{mM} \mathrm{Na} \mathrm{CO}_{3}$ and $0.05 \%$ Tween 80 . Bacterial suspensions were pipetted 10 times through a 26-gauge needle to remove $M$. tuberculosis aggregates. Latex beads with a diameter of $2.07 \mu \mathrm{m}$ (Bangs Laboratories) were labelled with FITC (Sigma-Aldrich) by incubation at $4{ }^{\circ} \mathrm{C}$ for 30 min with constant shaking at 700 r.p.m. in PBS, pH 8.3, containing $10 \mathrm{mM} \mathrm{Na}_{2} \mathrm{CO}_{3}$. Macrophages were infected at m.o.i. 50100 in triplicate. Phagocytosis was synchronized by allowing the bacteria to adhere to host cells for $60 \mathrm{~min}$ at $4{ }^{\circ} \mathrm{C}$ before starting phagocytosis by washing each well, adding preheated $\left(37^{\circ} \mathrm{C}\right)$ culture medium and incubating at $37{ }^{\circ} \mathrm{C}$ in $5 \% \mathrm{CO}_{2}$. After a chase of 5, 20, 60 or $1200 \mathrm{~min}$, coverslips were fixed with $4 \%$ paraformaldehyde in PBS, $\mathrm{pH}$ 7.4. Samples were incubated in PBS, pH 7.4, with $2 \%$ FCS, $0.2 \%$ $\mathrm{BSA}$ and $0.05 \%$ saponin prior to staining with the primary antibody rat anti-LAMP1 (Santa Cruz) and secondary antibody goat anti-rat Alexa Fluor 568 (Molecular Probes). Coverslips were mounted in Mowiol (Calbiochem), blinded and sequentially analysed for co-localization with a Leica TCS SP2 confocal microscope (Mannheim).

Measurement of intraphagosomal pH. Intraphagosomal pH measurement was performed as described previously (Master et al., 2008). Briefly, mycobacteria were labelled with 5(6)-carboxyfluorescein $\mathrm{N}$ succinimidyl ester (CFSE) (Sigma-Aldrich) by incubation in PBS, pH 7.4, containing $0.05 \%$ Tween 80 for $60 \mathrm{~min}$ and 5(6)-carboxyrhodamine 6G succinimidylester (CRSE) (Molecular Probes) for $30 \mathrm{~min}$ (added to the bacteria/CFSE suspension after $30 \mathrm{~min}$ ) at $37^{\circ} \mathrm{C}$ with shaking (400 r.p.m.) in the dark. Unreacted fluorophore was removed by washing with PBS, pH 7.4. Bacteria were added to triplicates of $2 \times 10^{5} \mathrm{ml}^{-1}$ host cells (BMDM or cell line J774.A1) at m.o.i. 100-500 in 96-well plates. Bacteria were sedimented to the cells by spin inoculation (300 r.p.m. for 5 min at $4{ }^{\circ} \mathrm{C}$ ) and incubated for $60 \mathrm{~min}$ at $4{ }^{\circ} \mathrm{C}$ for synchronization. Phagocytosis was initiated by washing each well, adding preheated culture medium and incubating at $37{ }^{\circ} \mathrm{C}$ in $5 \% \mathrm{CO}_{2}$. After 5 and $150 \mathrm{~min}$, cells were washed and maintained in $\mathrm{PBS}, \mathrm{pH} 7.4$, for recording with a spectrofluorimeter (Synergy HT, Bio-Tek). Intraphagosomal $\mathrm{pH}$ was calculated from CFSE/CRSE ratios using a calibration curve established from cells fed with double-labelled mycobacteria in PBS with defined $\mathrm{pH}$ (5.0-7.0) containing the ionophores nigericin $(20 \mu \mathrm{M})$ (Fluka) and monensin $(4 \mu \mathrm{M})$ (Fluka) to equilibrate the intracellular and extracellular $\mathrm{pH}$. Addition of bafilomycin Al $(40 \mu \mathrm{M})$ (Sigma-Aldrich) at the beginning of the incubation period dissipated the intraphagosomal $\mathrm{pH}$ measured at $150 \mathrm{~min}$, indicating that the signal originated from intracellular bacilli (data not shown).

\section{RESULTS}

\section{Attenuation of IspA : : aph does not involve TLR2}

M. tuberculosis lipoproteins trigger a TLR2 host response, which contributes to control of $M$. tuberculosis infection 
(Reiling et al., 2002) and phagosome maturation (Blander \& Medzhitov, 2006). We have recently shown that the lspA::aph mutant, which is impaired in lipoprotein synthesis, is severely attenuated in both resistant and susceptible mouse strains (Sander et al., 2004). To investigate the role of TLR2 in attenuation of the mutant, we infected $\mathrm{TLR}^{-1-}$ mice and mice of the isogenic parental strain C57BL/6 by aerosol with M. tuberculosis wild-type, $l s p A:: a p h$ mutant and $l s p A:: a p h$-lspA complemented mutant (Fig. 1). The lungs of mice infected with the parental strain exhibited the characteristic mode of bacterial growth: after initial multiplication during the first weeks, the number of c.f.u. reached a constant plateau over time. Following replication in the lungs the microorganisms disseminated into the spleen. As reported previously (Sander et al., 2004), the lspA::aph mutant showed a $2.5-3.5 \log$ reduction in c.f.u. in lungs (Fig. 1) and was markedly impaired in dissemination to the spleen (see Supplementary Fig. S1) compared with the wild-type. Complementation of the mutant restored the wild-type phenotype. For wild-type $M$. tuberculosis the bacterial burden of infected organs was similar in wild-type and TLR2 ${ }^{-l-}$ mice. Growth of the $\operatorname{lsp} A:: a p h$ mutant was likewise independent of the TLR2 genotype of the host, i.e. upon infection of $\mathrm{TLR}^{-1-}$ mice, the $\operatorname{lsp} A$ knockout mutant showed a similar reduction in c.f.u. in the lungs

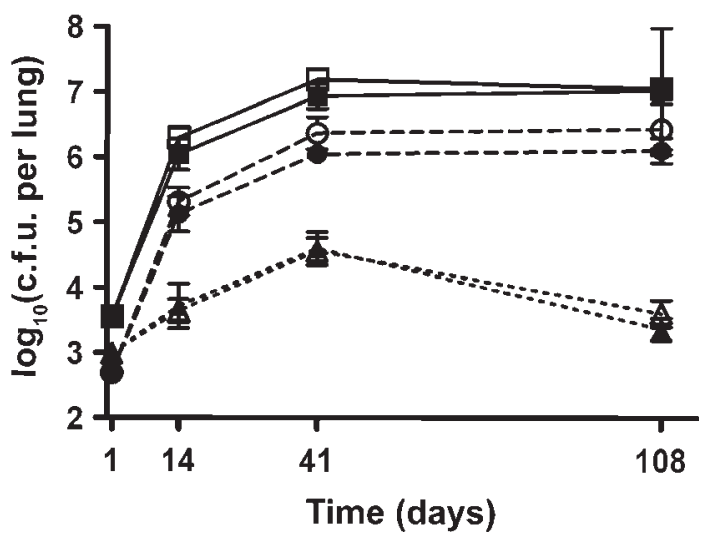

Fig. 1. Kinetics of in vivo growth of $M$. tuberculosis wild-type and mutants in lungs over a 108 day period. C57BL/6 mice (filled symbols) and C57BL/6 $\mathrm{TLR}^{-1-}$ mice (open symbols) were infected with $M$. tuberculosis wild-type (squares), IspA::aph mutant (triangles) and $I s p A:: a p h-I s p A$ complemented mutant (circles). The data are expressed as the mean \pm SD (error bars) of c.f.u. obtained with five mice at each time point. Data were analysed by one-way analysis of variance (ANOVA) followed by Bonferroni's multiple comparison test; $M$. tuberculosis wild type c.f.u. compared with /spA : : aph c.f.u. in C57BL/6 and in TLR2 ${ }^{-/-}$ mice were significantly different $(P<0.001)$; c.f.u. of $M$. tuberculosis wild type in C57BL/6 compared with c.f.u. in TLR2 ${ }^{-/-}$mice were not significantly different $(P>0.05)$; c.f.u. of $/ s p A:: a p h$ in C57BL/6 compared with c.f.u. in $\mathrm{TLR}^{-1-}$ mice were not significantly different $(P>0.05)$.
(Fig. 1) and impaired dissemination to the spleen (Supplementary Fig. S1) as upon infection of parental C57BL/6 mice. Complementation of $\operatorname{lsp} A:: a p h$ restored wild-type virulence in TLR2 ${ }^{-/-}$mice.

\section{IspA : : aph is attenuated in macrophages}

We next studied whether the in vivo attenuation of the lspA::aph mutant (Fig. 1; Sander et al., 2004) correlated with attenuation of the mutant in in vitro models of infection. We infected resting BMDM of BALB/c mice with M. tuberculosis wild-type or lspA::aph mutant. BMDM were lysed at different time points post-infection and plated on agar to count c.f.u. (Fig. 2a). c.f.u. of the wild type increased about 800 -fold over the time period studied, which is in the range reported by others for strain H37Rv in mouse BMDM (Berthet et al., 1998; Hunt et al., 2008; Rooyakkers \& Stokes, 2005). The increase in the number of c.f.u. was due to intracellular multiplication, as the bacteria in the cell medium account for less than $10 \%$ of the total bacterial counts (data not shown) and DMEM does not support mycobacterial replication (Bryk et al., 2008). The increase in c.f.u. for the $\operatorname{sp} A:: a p h$ mutant was approximately one-tenth of that of the wild type at day 9 after infection (Fig. 2a, inset). Activation of BMDM with IFN- $\gamma$ reduced intracellular multiplication of wild-type bacteria by 26 -fold. Likewise, the intracellular growth of the lspA::aph knockout mutant was impaired upon IFN- $\gamma$ activation (Fig. 2b). The relative difference in intracellular multiplication between the wild type and $\operatorname{lsp} A:: a p h$ mutant remained similar in resting and IFN- $\gamma$-activated BMDM ( 10-fold). Complementation restored wild-type growth (see Supplementary Fig. S2). The phenotype of in vitro infections paralleled the in vivo phenotype of the mutant, which already exhibited a growth defect in the initial phase of infection (Fig. 1). Multiplication in the mycobacterial growth media 7H9-ADC (see Supplementary Fig. S3) and 7H9-OADC (Sander et al., 2004) was not impaired.

\section{LspA and TNF- $\alpha$ secretion by BMDM}

Upon phagocytosis, M. tuberculosis induces TNF- $\alpha$ in macrophages, and host immunity against tuberculosis is crucially linked to this cytokine. An inverse correlation between TNF- $\alpha$ secretion and intracellular growth rate of clinical isolates has been reported (Theus et al., 2007). To study whether interference with lipoprotein maturation in $M$. tuberculosis directly or indirectly affects macrophage activation we infected BMDM with M. tuberculosis wildtype or $l s p A:: a p h$ mutant. We measured the amount of TNF- $\alpha$ released $5-72 \mathrm{~h}$ post-infection. Over the time period studied TNF- $\alpha$ secretion of both strains increased in parallel as compared with untreated controls, and there was no significant difference between wild-type and lspA::aph infected cells at any time (see Supplementary Fig. S4). Wild-type-like TNF- $\alpha$ secretion of BMDM upon infection with the $\operatorname{sp} A:: a p h$ mutant indicates that 
(a)

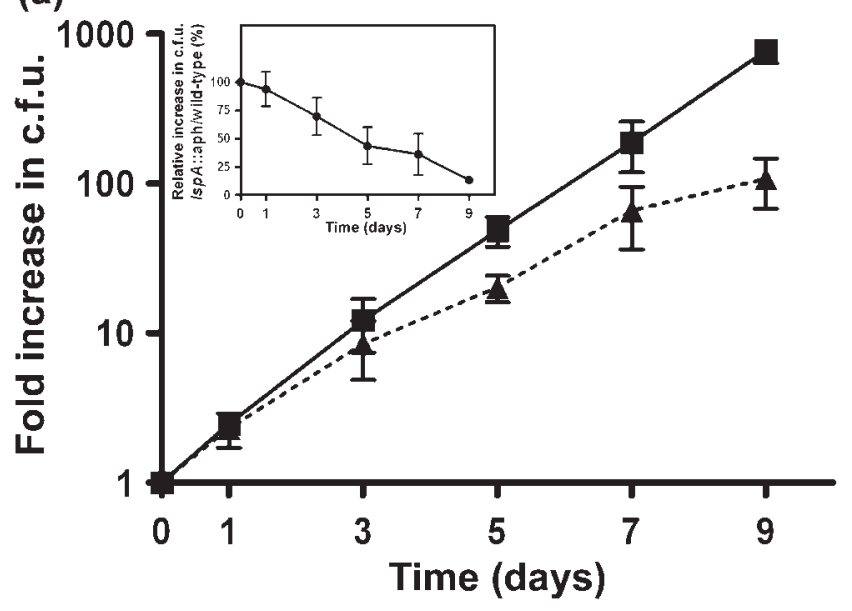

(b)

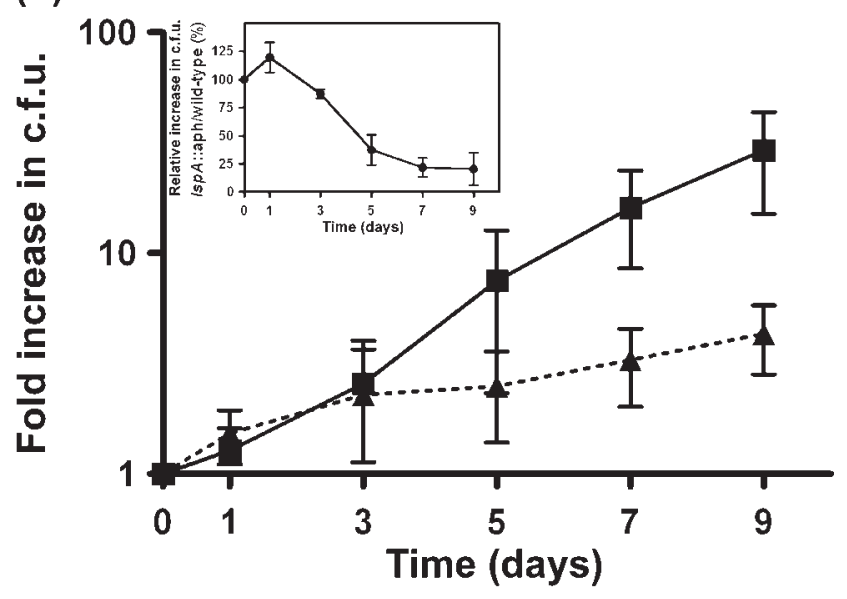

Fig. 2. Multiplication of $M$. tuberculosis wild-type and $/ s p A::$ aph mutant in (a) resting and (b) IFN- $\gamma$-activated BMDM at m.o.i. 0.5. Main panels show fold increase in c.f.u. of $M$. tuberculosis wildtype (回) and IspA : : aph mutant ( $(\boldsymbol{A})$. Insets show relative growth of IspA::aph mutant compared with wild-type as a percentage. Values represent the mean $\triangle S D$ (error bars) of four (resting BMDM) and three (activated BMDM) independent experiments. Statistical analysis was done by unpaired $t$ test; wild-type compared with IspA : : aph was significantly different $(P<0.05)$ at days 5,7 and 9 in unstimulated BMDM, and at days 7 and 9 in IFN$\gamma$-stimulated BMDM.

attenuation is not due to gross changes in macrophage bacterial sensing and signal transduction, corroborating the lack of TLR2-dependence evident in vivo.

\section{IspA : : aph phagosomes and LAMP1}

Phagosome maturation arrest is a striking feature of pathogenic mycobacteria and for some $M$. tuberculosis mutants a correlation between attenuation and loss of phagosome maturation arrest exists (Pethe et al., 2004). This prompted us to investigate whether the attenuated

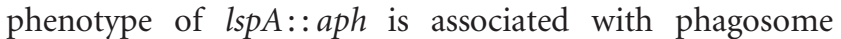
maturation. The phagosome acquires LAMP-1 during maturation, resulting in peak LAMP1 levels in late phagosomes and phago-lysosomes. We infected macrophages with fluorescently labelled latex beads, $M$. tuberculosis wild-type or $l s p A:: a p h$, and quantified co-localization of phagosomes with LAMP1 at different time points postinfection using confocal microscopy (Fig. 3a). Latex beads served as a positive control to demonstrate phagosome maturation. They rapidly and progressively co-localized with LAMP1 (Fig. 3c). Wild-type bacteria co-localized with LAMP1 to about $20 \%$ at 5, 20 and 60 min post-infection. Maximum co-localization of $\sim 40 \%$ was reached at the late time point $(20 \mathrm{~h})$, which is consistent with prior reports (Clemens \& Horwitz, 1995; Ramachandra et al., 2001; Xu et al., 1994). The lspA:: aph mutant showed wild-type-like characteristics with respect to degree and kinetics of LAMP1 co-localization (Fig. 3a, c).

IFN- $\gamma$ activation of macrophages promotes phagosome maturation in cells infected with $M$. tuberculosis. To study whether inactivation of LspA affects IFN- $\gamma$-mediated phagosome maturation, we quantified co-localization of mycobacterial phagosomes with LAMP1 in IFN- $\gamma$-activated BMDM (Fig. 3b). As early as $20 \mathrm{~min}$ after infection, $\sim 50 \%$ of $M$. tuberculosis wild-type phagosomes acquired LAMP1, and this percentage remained unchanged during the course of the experiment. The lspA::aph mutant phagosomes showed similar kinetics of LAMP1 acquisition to wild-type bacterial phagosomes (Fig. 3b, c). Thus, LspA inactivation has no effect on LAMP1 acquisition of M. tuberculosis-containing phagosomes in resting and activated macrophages.

\section{Acidification of IspA : : aph phagosomes}

We wanted to further characterize the $M$. tuberculosis $l s p A:: a p h$ phagosome and to corroborate the finding that phagosome maturation arrest is not affected by inactivation of LspA. Concurrent with phagosome maturation is acidification of the phagosomes (Crowle et al., 1991; Hackam et al., 1998; Oh \& Straubinger, 1996; SturgillKoszycki et al., 1994). Thus, we determined the intraphagosomal $\mathrm{pH}$ of $l s p A:$ aph phagosomes compared with $M$. tuberculosis wild-type phagosomes in BMDM by ratiometric measurement (Fig. 4a). The intraphagosomal pH was calculated from fluorescence ratio analysis after excitation at $485 / 530 \mathrm{~nm}$ and measurement at 528/ $590 \mathrm{~nm}$ with a spectrofluorimeter. The intraphagosomal $\mathrm{pH}$ of all strains started to decline within minutes, and at $150 \mathrm{~min}$ after infection wild-type phagosomes were modestly acidified with a mean $\mathrm{pH}$ of 6.4 , while phagosomes containing heat-inactivated wild-type bacteria were acidified to $\mathrm{pH}$ 5.8. These levels of intraphagosomal $\mathrm{pH}$ for live and dead mycobacteria are consistent with data reported elsewhere (de Chastellier \& Thilo, 2006; Russell et al., 2005; Sturgill-Koszycki et al., 1994). The $\mathrm{pH}$ of the lspA::aph phagosomes was 6.3, which is not significantly different from that of $M$. tuberculosis wild-type phago- 

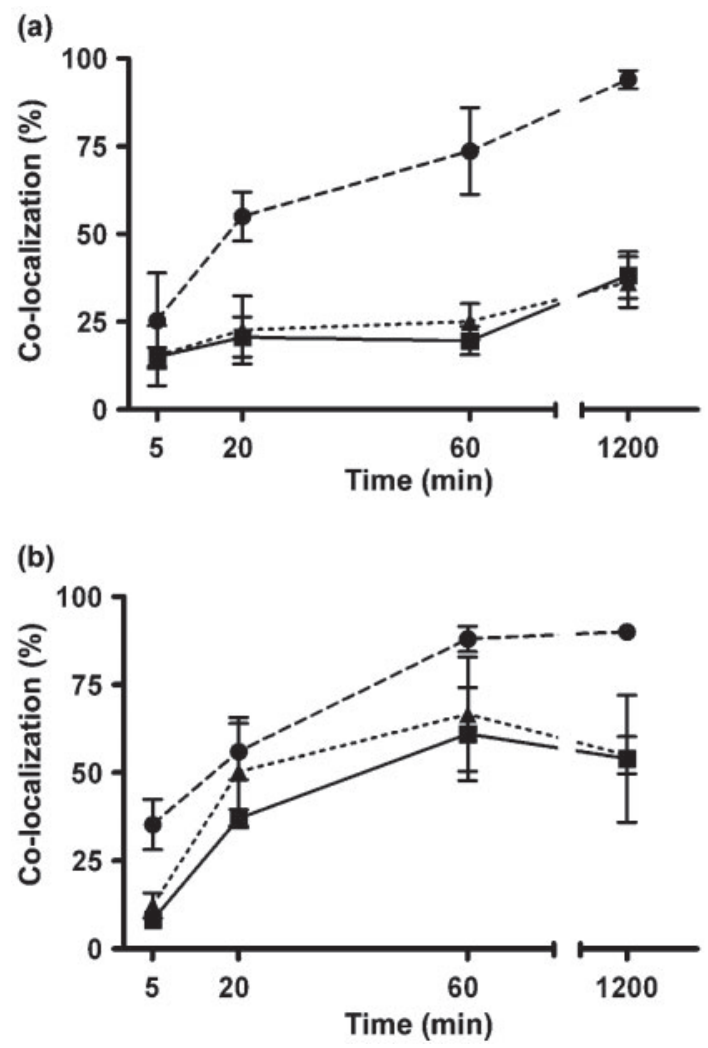

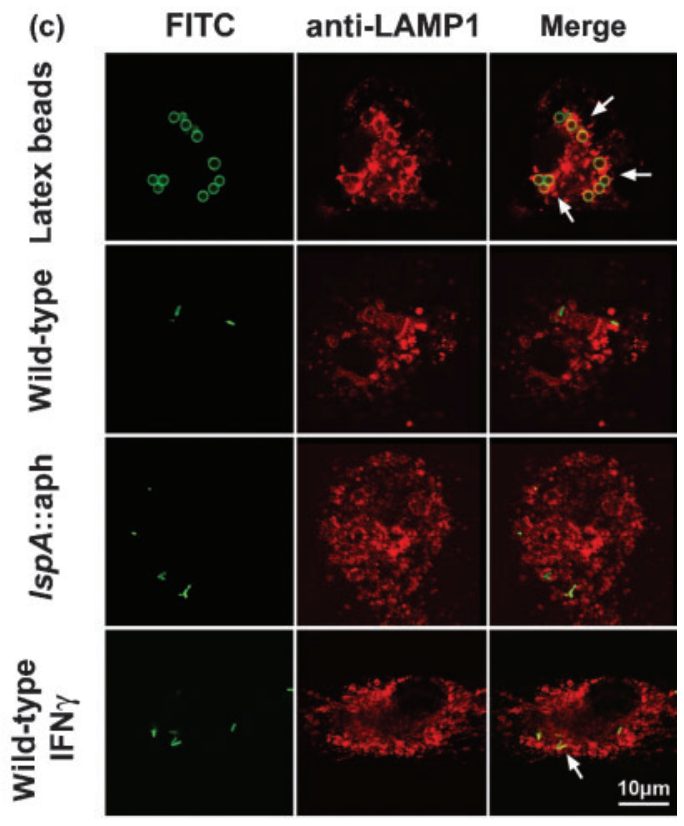

Fig. 3. Kinetics of co-localization of M. tuberculosis wild-type $(\boldsymbol{\square})$ and $/ s p A$ : : aph mutant $(\mathbf{\Delta})$ with late phagosomal marker LAMP1 in (a) resting and (b) IFN- $\gamma$-activated BMDM. Latex beads ( $)$ served as a positive control. Values represent the means \pm SD (error bars) of three independent experiments. Data were analysed by one-way ANOVA followed by Bonferroni's multiple comparison test; wild-type compared with $I s p A$ : : aph was not significantly different $(P>0.05)$. (c) Representative confocal immunofluorescence images are shown of non-activated macrophages infected with FITC-labelled latex beads (top row), M. tuberculosis wild-type (second row), IspA : : aph mutant (third row) or IFN- $\gamma$-activated macrophages infected with $M$. tuberculosis wild-type (bottom row) and immunostained for LAMP1 60 min after infection. Co-localization is indicated by arrows.

somes. Activation of BMDM with $\mathrm{IFN}-\gamma$ reduced the intraphagosomal $\mathrm{pH}$ to $\sim 5.7$, independent of the strain used for infection (Fig. 4b). Intraphagosomal $\mathrm{pH}$ measurements in unstimulated mouse monocyte-macrophage cell line J774A.1 revealed results similar to those in unstimulated BMDM: a significantly lower $\mathrm{pH}$ of phagosomes containing heat-killed mycobacteria as compared with live M. tuberculosis, and no difference between wild-type and the $\operatorname{lsp} A:$ : aph mutant (Fig. 4c).

\section{DISCUSSION}

We have previously shown that the $M$. tuberculosis lspA: : aph mutant has a strongly attenuated phenotype in a mouse infection model (Sander et al., 2004). Here we investigated putative mechanisms involved in attenuation of this mutant at a cellular level. We found that a TLR2dependent host response does not contribute to growth restriction of the $\operatorname{lsp} A:: a p h$ mutant. We also found that mature lipoproteins are dispensable for phagosome maturation arrest but required for intracellular survival. Our results demonstrate that intracellular survival of the $l s p A:$ aph mutant and phagosome maturation arrest are not necessarily linked but can be dissociated.

The M. tuberculosis genome encodes approximately 100 lipoproteins (Rezwan et al., 2007a; Sutcliffe \& Harrington, 2004). Some contribute to immunopathogenesis of tuberculosis via TLR2 (Bigi et al., 2004; Brightbill et al., 1999), while other lipoproteins have protective properties and are considered to be tuberculosis vaccine candidates (Romano et al., 2006; Wang et al., 2007). LspA mutants are unable to cleave off the signal peptide from pro-lipoproteins. The presence of a signal peptide inhibits the subsequent modification step and the transport of lipoproteins (Masuda et al., 2002). In LspA mutants, protein release (shedding) and proteolytic cleavage of immature lipoproteins (shaving) by proteases with other specificities has been reported (Antelmann et al., 2001). Both release and cleavage affect localization, function and antigenicity of proteins and may alter the protective or anti-protective 


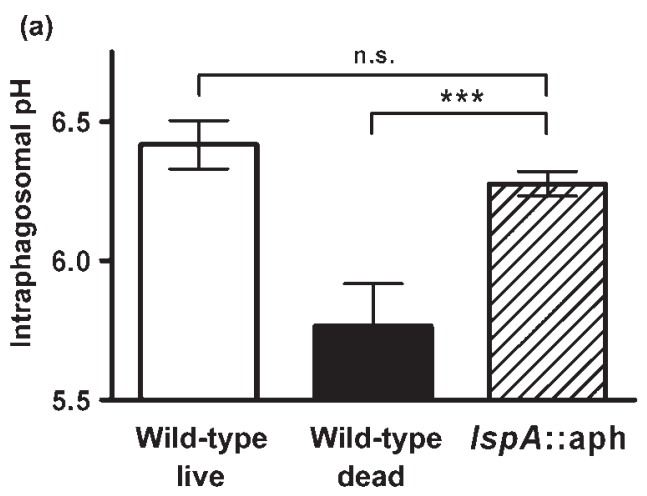

(b)
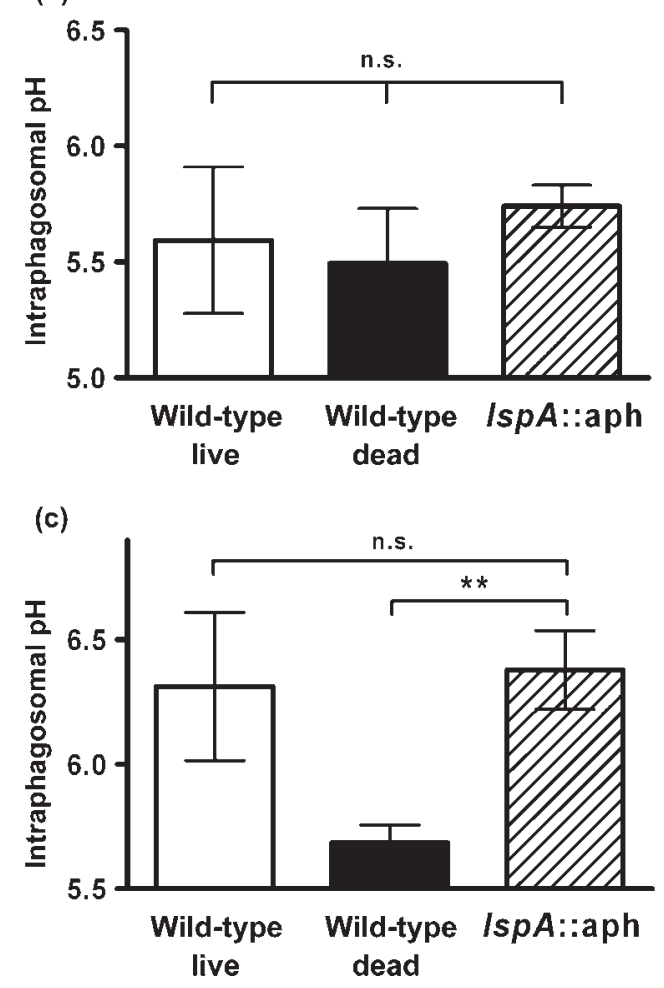

Fig. 4. Intraphagosomal $\mathrm{pH}$ of $M$. tuberculosis wild-type or IspA : : aph phagosomes in (a) resting BMDM, (b) IFN- $\gamma$-activated BMDM and (c) resting $\mathbf{J 7 7 4}$ at 150 min after infection. Values represent the mean $\mathrm{pH}$ of triplicates $\pm \mathrm{SD}$ (error bars) measured in six independent experiments. Dead (heat-inactivated) M. tuberculosis served as a control. Data were analysed by one-way ANOVA followed by Bonferroni's multiple comparison test; n.s., not significant $(P>0.05) ;{ }^{\star \star} P \leqslant 0.01 ;{ }^{\star \star \star} P<0.001$.

affect of lipoproteins. Under certain conditions, TLR2 contributes to control of M. tuberculosis infection (Reiling et al., 2002); therefore, we investigated growth of wild type and $l s p A:: a p h$ mutant strains in $\mathrm{TLR}^{-1-}$ and congenic C57BL/6 mice. We investigated virulence in a low-dose aerosol mouse model. Under these conditions the TLR2 genotype of the host does not affect control of $M$. tuberculosis wild-type (Fig. 1). Likewise, we observed attenuation of the lspA::aph mutant in $\mathrm{TLR} 2^{-1-}$ mice similar to that in congenic C57BL/6 mice. In addition, we measured a similar secretion of TNF- $\alpha$ by BMDM following in vitro infection with either mutant or wildtype (Supplementary Fig. S4). Thus, lipoprotein processing by LspA is not critical for TNF- $\alpha$ secretion or TLR2 responses to mycobacterial infection in vivo. Our in vitro findings are in agreement with those of others (Doz et al., 2007; Gilleron et al., 2003), who have reported that besides lipoproteins additional mycobacterial components such as PIM activate TLR2 in primary macrophages. In contrast, Banaiee et al. (2006) found a modestly reduced TNF- $\alpha$ secretion in RAW264.7 cells and defective TLR2 stimulation in HEK293-TLR2 reporter cells when exposed to a $\Delta l s p A$ knockout mutant. This discrepancy may be explained by the different experimental settings. Our in vitro and in vivo data effectively rule out a significant involvement of TLR2 in virulence attenuation of the lspA: : aph mutant.

A number of studies demonstrate a correlation between in vivo attenuation of mycobacterial mutants and impaired in vitro survival in macrophages (Camacho et al., 1999; Sassetti \& Rubin, 2003). The in vivo phenotype of the lspA::aph mutant is characterized by a pronounced reduction in c.f.u., which is already prominent soon after infection. We therefore studied whether this was concurrent with a reduced survival in macrophages. Macrophages are major effector cells involved in control of bacterial infection, but are also a major niche for multiplication of pathogenic mycobacteria. In in vitro macrophage infection experiments a variety of $M$. tuberculosis growth/survival rates, ranging from complete growth restriction (Vandal et al., 2006) to extensive multiplication (100-fold in 6 days; 1000-fold in 9 days) (Berthet et al., 1998; Rooyakkers \& Stokes, 2005), have been described. In our studies, $M$. tuberculosis multiplied $\sim 800$-fold over 9 days. Stimulation of macrophages with IFN- $\gamma$ consistently restricts growth of M. tuberculosis in relation to unstimulated cells. Again, huge differences exist in the literature with respect to absolute growth rates in IFN- $\gamma$-activated cells, ranging from $90 \%$ killing (MacMicking et al., 2003) to more than a 20-fold increase in 7 days (Rooyakkers \& Stokes, 2005). In our experiments IFN- $\gamma$ reduced growth of $M$. tuberculosis as compared with untreated controls 26-fold, but still allowed multiplication of $M$. tuberculosis. The reasons for the inter-laboratory growth differences of $M$. tuberculosis in both unstimulated and stimulated macrophages are not entirely clear. Compared with $M$. tuberculosis wild-type the growth of the mutant was significantly reduced in resting as well as in IFN- $\gamma$-activated BMDM (Fig. 2). Wild-type and $l s p A:: a p h$ show an identical growth rate in culture broth (Supplementary Fig. S3), excluding a simple growth deficit of the $l s p A:$ aph mutant. Thus, inactivation of LspA causes an intracellular fitness reduction of the bacteria upon both in vivo and in vitro infection.

The ability to arrest phagosome maturation is a hallmark of M. tuberculosis (Armstrong \& Hart, 1971). Besides 
avoidance of phago-lysosomal fusion, relatively little is known about how $M$. tuberculosis manages its intracellular lifestyle. Few of the various M. tuberculosis mutants which exhibit in vivo attenuation (Sassetti \& Rubin, 2003; Smith, 2003) have been characterized with respect to intracellular localization. Likewise few of the mutants displaying a trafficking phenotype have been characterized for in vivo attenuation (MacGurn \& Cox, 2007; Pethe et al., 2004). While lipids of the mycobacterial cell wall are reportedly involved in phagosome maturation arrest, the role of lipoproteins is less clear. When studying co-localization of wild-type and $\operatorname{spA}:: a p h$ mutant bacteria with the phagosomal/phagolysosomal marker LAMP1 we found little colocalization for both strains in resting macrophages (Fig. 3). In contrast, a mutant deficient in zinc metalloprotease $z m p 1$ shows a high degree of co-localization (Master et al., 2008). To corroborate $\operatorname{lsp} A:: a p h$ localization in an early, i.e. a LAMP-negative, phagosome we determined phagosomal $\mathrm{pH}$, which is considered to be the ultimate marker for phagosome maturation (Huynh \& Grinstein, 2007). We measured intraphagosomal $\mathrm{pH}$ of wild-type and $\operatorname{lspA}:: a p h$ mutant phagosomes (Fig. 4). The intraphagosomal $\mathrm{pH}$ was $\sim 6.4$ for both strains, demonstrating that there is no apparent difference between the $\operatorname{sp} A:: a p h$ phagosome and the $M$. tuberculosis wild-type phagosome. Thus, both strains localize to a compartment with early endosomal characteristics, including absence of LAMP1 and mildly acidic $\mathrm{pH}$. In contrast, phagosomes containing zmpl mutants show a reduced $\mathrm{pH}$ (Master et al., 2008). Following IFN- $\gamma$ activation of the host cell, the mycobacteria-containing compartment acidifies to a $\mathrm{pH}$ of about 5.7. IFN- $\gamma$-induced acidification was also found in cells infected with the $\operatorname{lsp} A:: a p h$ mutant and was similar to that in cells infected with the wild-type strain. We conclude that inactivation of LspA does not affect phagosome-maturation arrest, indicating that attenuation of the mutant takes place in a prelysosomal compartment. This suggests that LspA is required for multiplication of $M$. tuberculosis in early phagosomes. The early phagosome is thought to be a nutrient-restricted environment, which is important in limiting the availability of nutients to phagocytosed bacteria (De Voss et al., 2000; Wagner et al., 2005). Despite some research in this field the key elements critical for adaptation of mycobacteria to this prelysosomal environment are unknown.

Approximately $100 \mathrm{M}$. tuberculosis proteins have been annotated as lipoproteins. Several of these lipoproteins are involved in uptake of nutrients or ions (PstS, ModA, FecB, LpqZ, LpqY; Sutcliffe \& Harrington, 2004). Thus, the limited growth of the lspA::aph mutant in the nutrientpoor phagosome may be due to mislocation or malfunction of transport-associated lipoproteins. These uptake mechanisms may only be important in the nutrient-limited milieu of the early phagosome and not in nutrient-rich broth medium. Alternatively, the $l s p A:: a p h$ mutant may be more susceptible to direct host attacks. The physiology of $M$. tuberculosis is intimately linked to integrity of the cell wall, and some lipoproteins are involved in cell wall metabolism
(LppX, LpqK, LppW; Sutcliffe \& Harrington, 2004). LppX is required for the translocation of phthiocerol dimycocerosates to the outer membrane of $M$. tuberculosis, and disruption of $\operatorname{lp} p X$ is accompanied by attenuation of the tubercle bacillus (Sulzenbacher et al., 2006). At a gross level, i.e. by Ziehl-Neelsen staining, mycolic acid analysis and inspection by electron microscopy (Sander et al., 2004), the cell wall of the $\operatorname{lsp} A$ mutant is indistinguishable from that of the parental strain. However, minor alterations not detected by these investigations may increase the susceptibility of the mutant to antibacterial mechanisms of the host. Finally, lipoproteins of unknown functions (e.g. Mpt83) which are highly upregulated during all stages of in vitro and in vivo infection (Schnappinger et al., 2003) may be required for intracellular survival and multiplication.

Deciphering the M. tuberculosis genome has facilitated the development of screens designed to identify genes implicated in the infection process (Lamichhane et al., 2003; Sassetti et al., 2001; Sassetti \& Rubin, 2003). Overall, a significant overlap between genes required for survival of $M$. tuberculosis in macrophages and those required for full-blown virulence in in vivo infection of mice has been found. With respect to a correlation between intracellular survival and phagosome acidification, the results from genetic screens are contradictory and inconsistent. In a Mycobacterium bovis BCG transposon library screen designed to identify mutants defective in intracellular survival or in prevention of phagosomal acidification, little overlap between the two phenotypes was observed (Stewart et al., 2005). Similarly, MacGurn \& Cox (2007) observed little correlation between the degree of attenuation in vivo and phagosome maturation of the screened mutants. In contrast, the transposon mutagenesis screen reported by Pethe et al. (2004) found a correlation between mutants with increased localization to lysosomes and mutants with reduced intracellular survival (Pethe et al., 2004). Surprisingly, no matches among the mutants identified to be deficient in phagosome maturation arrest exist between the three screens. In addition, neither screen picked up factors known to be involved in phagosome maturation arrest, e.g. lipoarabinomannan (LAM).

Phagosome maturation arrest is an active process requiring live bacteria. Our findings disprove the hypothesis that lspA:: aph attenuation in vivo parallels phagosome acidification. Our results point to a mechanism restricting intracellular growth of $M$. tuberculosis that acts prior to the conversion of early phagosomes to late phagosomes. They indicate a role for the early mycobacterial phagosome in growth restriction of intracellular M. tuberculosis and of non-killing mechanisms in the control of tuberculosis infection, mechanisms that are unrelated to phagosome maturation arrest and avoidance of phago-lysosome fusion.

\section{ACKNOWLEDGEMENTS}

We thank Thomas Bächi and Mathias Höchli (Laboratory of Electron Microscopy, University of Zurich) for help with confocal microscopy. The original breeding pairs of TLR $2^{-1-}$ mice were kindly provided by 
Carsten Kirschning, Technical University of Munich, Germany. This work was supported in part by the Swiss National Science Foundation (3200-068488; 3100AO_120326) to E.C.B. and P.S., EU grant TB-Drug (LSHP-CT-2006-037217) to P.S., a grant from the Niedersächsischer Verein zur Bekämpfung der Tuberkulose Lungenund Bronchialerkrankungen e.V. to E.C.B., and by DFG grant SFB470C9 to S.E. S. K. R. is supported by grants from Swiss Lung Foundation, Wolfermann-Nägeli Foundation, OPO-Pharma Foundation and Frieda Locher Foundation. The authors have no conflict of interest to declare.

\section{REFERENCES}

Anes, E., Kuhnel, M. P., Bos, E., Moniz-Pereira, J., Habermann, A. \& Griffiths, G. (2003). Selected lipids activate phagosome actin assembly and maturation resulting in killing of pathogenic mycobacteria. Nat Cell Biol 5, 793-802.

Antelmann, H., Tjalsma, H., Voigt, B., Ohlmeier, S., Bron, S., van Dijl, J. M. \& Hecker, M. (2001). A proteomic view on genome-based signal peptide predictions. Genome Res 11, 1484-1502.

Armstrong, J. A. \& Hart, P. D. (1971). Response of cultured macrophages to Mycobacterium tuberculosis, with observations on fusion of lysosomes with phagosomes. J Exp Med 134, 713-740.

Banaiee, N., Kincaid, E. Z., Buchwald, U., Jacobs, W. R., Jr \& Ernst, J. D. (2006). Potent inhibition of macrophage responses to IFN- $\gamma$ by live virulent Mycobacterium tuberculosis is independent of mature mycobacterial lipoproteins but dependent on TLR2. J Immunol 176, 3019-3027.

Berthet, F. X., Lagranderie, M., Gounon, P., Laurent-Winter, C., Ensergueix, D., Chavarot, P., Thouron, F., Maranghi, E., Pelicic, V. \& other authors (1998). Attenuation of virulence by disruption of the Mycobacterium tuberculosis erp gene. Science 282, 759-762.

Bigi, F., Gioffre, A., Klepp, L., Santangelo, M. P., Alito, A., Caimi, K., Meikle, V., Zumárraga, M., Taboga, O. \& other authors (2004). The

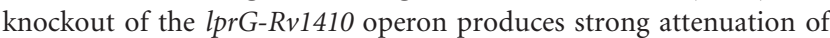
Mycobacterium tuberculosis. Microbes Infect 6, 182-187.

Blander, J. M. \& Medzhitov, R. (2004). Regulation of phagosome maturation by signals from Toll-like receptors. Science 304, 1014-1018.

Blander, J. M. \& Medzhitov, R. (2006). On regulation of phagosome maturation and antigen presentation. Nat Immunol 7, 1029-1035.

Brightbill, H. D., Libraty, D. H., Krutzik, S. R., Yang, R. B., Belisle, J. T., Bleharski, J. R., Maitland, M., Norgard, M. V., Plevy, S. E. \& other authors (1999). Host defense mechanisms triggered by microbial lipoproteins through Toll-like receptors. Science 285, 732-736.

Bryk, R., Gold, B., Venugopal, A., Sing, J., Samy, R., Pupek, K., Cao, H., Popescu, C., Gurney, M. \& other authors (2008). Selective killing of nonreplicating mycobacteria. Cell Host Microbe 3, 137-145.

Camacho, L. R., Ensergueix, D., Perez, E., Gicquel, B. \& Guilhot, C. (1999). Identification of a virulence gene cluster of Mycobacterium tuberculosis by signature-tagged transposon mutagenesis. Mol Microbiol 34, 257-267.

Chua, J., Vergne, I., Master, S. \& Deretic, V. (2004). A tale of two lipids: Mycobacterium tuberculosis phagosome maturation arrest. Curr Opin Microbiol 7, 71-77.

Clemens, D. L. \& Horwitz, M. A. (1995). Characterization of the Mycobacterium tuberculosis phagosome and evidence that phagosomal maturation is inhibited. J Exp Med 181, 257-270.

Clemens, D. L. \& Horwitz, M. A. (1996). The Mycobacterium tuberculosis phagosome interacts with early endosomes and is accessible to exogenously administered transferrin. J Exp Med 184, 1349-1355.
Clemens, D. L., Lee, B. Y. \& Horwitz, M. A. (2000). Mycobacterium tuberculosis and Legionella pneumophila phagosomes exhibit arrested maturation despite acquisition of Rab7. Infect Immun 68, 5154-5166.

Crowle, A. J., Dahl, R., Ross, E. \& May, M. H. (1991). Evidence that vesicles containing living, virulent Mycobacterium tuberculosis or Mycobacterium avium in cultured human macrophages are not acidic. Infect Immun 59, 1823-1831.

de Chastellier, C. \& Thilo, L. (2006). Cholesterol depletion in Mycobacterium avium-infected macrophages overcomes the block in phagosome maturation and leads to the reversible sequestration of viable mycobacteria in phagolysosome-derived autophagic vacuoles. Cell Microbiol 8, 242-256.

Dev, I. K. \& Ray, P. H. (1984). Rapid assay and purification of a unique signal peptidase that processes the prolipoprotein from Escherichia coli B. J Biol Chem 259, 11114-11120.

De Voss, J. J., Rutter, K., Schroeder, B. G., Su, H., Zhu, Y. \& Barry, C. E., III (2000). The salicylate-derived mycobactin siderophores of Mycobacterium tuberculosis are essential for growth in macrophages. Proc Natl Acad Sci U S A 97, 1252-1257.

Doz, E., Rose, S., Nigou, J., Gilleron, M., Puzo, G., Erard, F., Ryffel, B. \& Quesniaux, V. F. J. (2007). Acylation determines the Toll-like receptor (TLR)-dependent positive versus TLR2-, mannose receptor-, and SIGNR1-independent negative regulation of pro-inflammatory cytokines by mycobacterial lipomannan. J Biol Chem 282, 2601426025.

Fratti, R. A., Backer, J. M., Gruenberg, J., Corvera, S. \& Deretic, V. (2001). Role of phosphatidylinositol 3-kinase and Rab5 effectors in phagosomal biogenesis and mycobacterial phagosome maturation arrest. J Cell Biol 154, 631-644.

Fratti, R. A., Chua, J., Vergne, I. \& Deretic, V. (2003). Mycobacterium tuberculosis glycosylated phosphatidylinositol causes phagosome maturation arrest. Proc Natl Acad Sci U S A 100, 5437-5442.

Gehring, A. J., Dobos, K. M., Belisle, J. T., Harding, C. V. \& Boom, W. H. (2004). Mycobacterium tuberculosis LprG (Rv1411c): a novel TLR-2 ligand that inhibits human macrophage class II MHC antigen processing. J Immunol 173, 2660-2668.

Gilleron, M., Quesniaux, V. F. J. \& Puzo, G. (2003). Acylation state of the phosphatidylinositol hexamannosides from Mycobacterium bovis bacillus Calmette Guerin and Mycobacterium tuberculosis H37Rv and its implication in Toll-like receptor response. J Biol Chem 278, 2988029889 .

Hackam, D. J., Rotstein, O. D., Zhang, W., Gruenheid, S., Gros, P. \& Grinstein, S. (1998). Host resistance to intracellular infection: mutation of natural resistance-associated macrophage protein 1 (Nramp1) impairs phagosomal acidification. J Exp Med 188, 351-364.

Hasan, Z., Schlax, C., Kuhn, L., Lefkovits, I., Young, D., Thole, J. \& Pieters, J. (1997). Isolation and characterization of the mycobacterial phagosome: segregation from the endosomal/lysosomal pathway. Mol Microbiol 24, 545-553.

Hostetter, J. M., Steadham, E. M., Haynes, J. S., Bailey, T. B. \& Cheville, N. F. (2002). Cytokine effects on maturation of the phagosomes containing Mycobacterium avium subspecies paratuberculosis in J774 cells. FEMS Immunol Med Microbiol 34, 127-134.

Hunt, D. M., Saldanha, J. W., Brennan, J. F., Benjamin, P., Strom, M., Cole, J. A., Spreadburry, C. L. \& Buxton, R. S. (2008). Single nucleotide polymorphisms that cause structural changes in the cyclic AMP receptor protein transcriptional regulator of the tuberculosis vaccine strain Mycobacterium bovis BCG alter global gene expression without attenuating growth. Infect Immun 76, 2227-2234.

Huynh, K. K. \& Grinstein, S. (2007). Regulation of vacuolar pH and its modulation by some microbial species. Microbiol Mol Biol Rev 71, $452-462$. 
Indrigo, J., Hunter, R. L., Jr \& Actor, J. K. (2003). Cord factor trehalose 6,6'-dimycolate (TDM) mediates trafficking events during mycobacterial infection of murine macrophages. Microbiology 149, 2049-2059.

Jin, M. S., Kim, E., Heo, J. Y., Lee, M. E., Kim, H. M., Paik, S. G., Lee, H. \& Lee, J. O. (2007). Crystal structure of the TLR1-TLR2 heterodimer induced by binding of tri-acylated lipopeptide. Cell 130, 1071-1082.

Kanzler, H., Barrat, F. J., Hessel, E. M. \& Coffman, R. L. (2007). Therapeutic targeting of innate immunity with Toll-like receptor agonists and antagonists. Nat Med 13, 552-559.

Kelley, V. A. \& Schorey, J. S. (2003). Mycobacterium's arrest of phagosome maturation in macrophages requires Rab5 activity and accessibility to iron. Mol Biol Cell 14, 3366-3377.

Lamichhane, G., Zignol, M., Blades, N. J., Geiman, D. E., Dougherty, A., Grosset, J., Broman, K. W. \& Bishai, W. R. (2003). A postgenomic method for predicting essential genes at subsaturation levels of mutagenesis: application to Mycobacterium tuberculosis. Proc Natl Acad Sci U S A 100, 7213-7218.

MacGurn, J. A. \& Cox, J. S. (2007). A genetic screen for Mycobacterium tuberculosis mutants defective for phagosome maturation arrest identifies components of the ESX-1 secretion system. Infect Immun 75, 2668-2678.

MacMicking, J. D., Taylor, G. A. \& McKinney, J. D. (2003). Immune control of tuberculosis by IFN- $\gamma$-inducible LRG-47. Science 302, 654659 .

Master, S. S., Rampini, S. K., Davis, A. S., Keller, C., Ehlers, S., Springer, B., Timmins, G., Sander, P. \& Deretic, V. (2008). Mycobacterium tuberculosis prevents inflammasome activation. Cell Host Microbe 3, 224-232.

Masuda, K., Matsuyama, S. \& Tokuda, H. (2002). Elucidation of the function of lipoprotein-sorting signals that determine membrane localization. Proc Natl Acad Sci U S A 99, 7390-7395.

Mawuenyega, K. G., Forst, C. V., Dobos, K. M., Belisle, J. T., Chen, J., Bradbury, E. M., Bradbury, A. R. \& Chen, X. (2005). Mycobacterium tuberculosis functional network analysis by global subcellular protein profiling. Mol Biol Cell 16, 396-404.

Noss, E. H., Pai, R. K., Sellati, T. J., Radolf, J. D., Belisle, J., Golenbock, D. T., Boom, W. H. \& Harding, C. V. (2001). Toll-like receptor 2-dependent inhibition of macrophage class II MHC expression and antigen processing by $19-\mathrm{kDa}$ lipoprotein of Mycobacterium tuberculosis. J Immunol 167, 910-918.

Oh, Y. K. \& Straubinger, R. M. (1996). Intracellular fate of Mycobacterium avium: use of dual-label spectrofluorometry to investigate the influence of bacterial viability and opsonization on phagosomal $\mathrm{pH}$ and phagosome-lysosome interaction. Infect Immun 64, 319-325.

Pethe, K., Swenson, D. L., Alonso, S., Anderson, J., Wang, C. \& Russell, D. G. (2004). Isolation of Mycobacterium tuberculosis mutants defective in the arrest of phagosome maturation. Proc Natl Acad Sci U S A 101, 13642-13647.

Ramachandra, L., Noss, E., Boom, W. H. \& Harding, C. V. (2001). Processing of Mycobacterium tuberculosis antigen 85B involves intraphagosomal formation of peptide-major histocompatibility complex II complexes and is inhibited by live bacilli that decrease phagosome maturation. J Exp Med 194, 1421-1432.

Reiling, N., Holscher, C., Fehrenbach, A., Kroger, S., Kirschning, C. J., Goyert, S. \& Ehlers, S. (2002). Cutting edge: Toll-like receptor (TLR)2- and TLR4-mediated pathogen recognition in resistance to airborne infection with Mycobacterium tuberculosis. J Immunol 169, 3480-3484.

Rezwan, M., Grau, T., Tschumi, A. \& Sander, P. (2007a). Lipoprotein synthesis in mycobacteria. Microbiology 153, 652-658.
Rezwan, M., Laneelle, M. A., Sander, P. \& Daffé, M. (2007b). Breaking down the wall: fractionation of mycobacteria. J Microbiol Methods 68, 32-39.

Rock, F. L., Hardiman, G., Timans, J. C., Kastelein, R. A. \& Bazan, J. F. (1998). A family of human receptors structurally related to Drosophila Toll. Proc Natl Acad Sci U S A 95, 588-593.

Romano, M., Roupie, V., Wang, X. M., Denis, O., Jurion, F., Adnet, P. Y., Laali, R. \& Huygen, K. (2006). Immunogenicity and protective efficacy of tuberculosis DNA vaccines combining mycolyl-transferase Ag85A and phosphate transport receptor PstS-3. Immunology 118, 321-332.

Rooyakkers, A. W. J. \& Stokes, R. W. (2005). Absence of complement receptor 3 results in reduced binding and ingestion of Mycobacterium tuberculosis but has no significant effect on the induction of reactive oxygen and nitrogen intermediates or on the survival of the bacteria in resident and interferon-gamma activated macrophages. Microb Pathog 39, 57-67.

Russell, D. G. (2001). Mycobacterium tuberculosis: here today, and here tomorrow. Nat Rev Mol Cell Biol 2, 569-577.

Russell, D. G., Purdy, G. E., Owens, R. M., Rohde, K. H. \& Yates, R. M. (2005). Mycobacterium tuberculosis and the four-minute phagosome. ASM News 71, 459-463.

Saleh, M. T. \& Belisle, J. T. (2000). Secretion of an acid phosphatase (SapM) by Mycobacterium tuberculosis that is similar to eukaryotic acid phosphatases. J Bacteriol 182, 6850-6853.

Sander, P., Rezwan, M., Walker, B., Rampini, S. K., Kroppenstedt, R. M., Ehlers, S., Keller, C., Keeble, J. R., Hagemeier, M. \& other authors (2004). Lipoprotein processing is required for virulence of Mycobacterium tuberculosis. Mol Microbiol 52, 1543-1552.

Sankaran, K. \& Wu, H. C. (1994). Lipid modification of bacterial prolipoprotein: transfer of diacylglyceryl moiety from phosphatidylglycerol. J Biol Chem 269, 19701-19706.

Sassetti, C. M. \& Rubin, E. J. (2003). Genetic requirements for mycobacterial survival during infection. Proc Natl Acad Sci U S A 100, 12989-12994.

Sassetti, C. M., Boyd, D. H. \& Rubin, E. J. (2001). Comprehensive identification of conditionally essential genes in mycobacteria. Proc Natl Acad Sci U S A 98, 12712-12717.

Schaible, U. E., Sturgill-Koszycki, S., Schlesinger, P. H. \& Russell, D. G. (1998). Cytokine activation leads to acidification and increases maturation of Mycobacterium avium-containing phagosomes in murine macrophages. J Immunol 160, 1290-1296.

Schnappinger, D., Ehrt, S., Voskuil, M. I., Liu, Y., Mangan, J. A., Monahan, I. M., Dolganov, G., Efron, B., Butcher, P. D. \& other authors (2003). Transcriptional adaptation of Mycobacterium tuberculosis within macrophages: insights into the phagosomal environment. J Exp Med 198, 693-704.

Smith, I. (2003). Mycobacterium tuberculosis pathogenesis and molecular determinants of virulence. Clin Microbiol Rev 16, 463-496.

Stewart, G. R., Patel, J., Robertson, B. D., Rae, A. \& Young, D. B. (2005). Mycobacterial mutants with defective control of phagosomal acidification. PLoS Pathog 1, 269-278.

Sturgill-Koszycki, S., Schlesinger, P. H., Chakraborty, P., Haddix, P. L., Collins, H. L., Fok, A. K., Allen, R. D., Gluck, S. L., Heuser, J. \& Russell, D. G. (1994). Lack of acidification in Mycobacterium phagosomes produced by exclusion of the vesicular proton-ATPase. Science 263, 678-681.

Sturgill-Koszycki, S., Schaible, U. E. \& Russell, D. G. (1996). Mycobacterium-containing phagosomes are accessible to early endosomes and reflect a transitional state in normal phagosome biogenesis. EMBO J 15, 6960-6968. 
Sulzenbacher, G., Canaan, S., Bordat, Y., Neyrolles, O., Stadthagen, G., Roig-Zamboni, V., Rauzier, J., Maurin, D., Laval, F. \& other authors (2006). LppX is a lipoprotein required for the translocation of phthiocerol dimycocerosates to the surface of Mycobacterium tuberculosis. EMBO J 25, 1436-1444.

Sutcliffe, I. C. \& Harrington, D. J. (2004). Lipoproteins of Mycobacterium tuberculosis: an abundant and functionally diverse class of cell envelope components. FEMS Microbiol Rev 28, 645-659.

Sutcliffe, I. C. \& Russell, R. R. (1995). Lipoproteins of Gram-positive bacteria. J Bacteriol 177, 1123-1128.

Theus, S., Eisenach, K., Fomukong, N., Silver, R. F. \& Cave, M. D. (2007). Beijing family Mycobacterium tuberculosis strains differ in their intracellular growth in THP-1 macrophages. Int J Tuberc Lung Dis 11, 1087-1093.

Underhill, D. M., Ozinsky, A., Hajjar, A. M., Stevens, A., Wilson, C. B., Bassetti, M. \& Aderem, A. (1999). The Toll-like receptor 2 is recruited to macrophage phagosomes and discriminates between pathogens. Nature 401, 811-815.

Vandal, O. H., Gelb, M. H., Ehrt, S. \& Nathan, C. (2006). Cytosolic phospholipase A2 enzymes are not required by mouse bone marrowderived macrophages for the control of Mycobacterium tuberculosis in vitro. Infect Immun 74, 1751-1756.

Vergne, I., Chua, J. \& Deretic, V. (2003). Tuberculosis toxin blocking phagosome maturation inhibits a novel $\mathrm{Ca}^{2+} /$ calmodulin-PI3K hVPS34 cascade. J Exp Med 198, 653-659.

Vergne, I., Fratti, R. A., Hill, P. J., Chua, J., Belisle, J. \& Deretic, V. (2004). Mycobacterium tuberculosis phagosome maturation arrest: mycobacterial phosphatidylinositol analog phosphatidylinositol mannoside stimulates early endosomal fusion. Mol Biol Cell 15, 751-760.

Vergne, I., Chua, J., Lee, H.-H., Lucas, M., Belisle, J. \& Deretic, V. (2005). Mechanism of phagolysosome biogenesis block by viable
Mycobacterium tuberculosis. Proc Natl Acad Sci U S A 102, 40334038.

Via, L. E., Deretic, D., Ulmer, R. J., Hibler, N. S., Huber, L. A. \& Deretic, V. (1997). Arrest of mycobacterial phagosome maturation is caused by a block in vesicle fusion between stages controlled by rab5 and rab7. J Biol Chem 272, 13326-13331.

Via, L. E., Fratti, R. A., McFalone, M., Pagan-Ramos, E., Deretic, D. \& Deretic, V. (1998). Effects of cytokines on mycobacterial phagosome maturation. J Cell Sci 111, 897-905.

Vieira, O. V., Botelho, R. J. \& Grinstein, S. (2002). Phagosome maturation: aging gracefully. Biochem J 366, 689-704.

Wagner, D., Maser, J., Moric, I., Boechat, N., Vogt, S., Gicquel, B., Lai, B., Reyrat, J. M. \& Bermudez, L. (2005). Changes of the phagosomal elemental concentrations by Mycobacterium tuberculosis Mramp. Microbiology 151, 323-332.

Walburger, A., Koul, A., Ferrari, G., Nguyen, L., PrescianottoBaschong, C., Huygen, K., Klebl, B., Thompson, C., Bacher, G. \& Pieters, J. (2004). Protein kinase $G$ from pathogenic mycobacteria promotes survival within macrophages. Science 304, 1800-1804.

Wang, B., Henao-Tamayo, M., Harton, M., Ordway, D., Shanley, C., Basaraba, R. J. \& Orme, I. M. (2007). A Toll-like receptor-2-directed fusion protein vaccine against tuberculosis. Clin Vaccine Immunol 14, 902-906.

Xu, S., Cooper, A., Sturgill-Koszycki, S., van Heyningen, T., Chatterjee, D., Orme, I., Allen, P. \& Russell, D. G. (1994). Intracellular trafficking in Mycobacterium tuberculosis and Mycobacterium avium-infected macrophages. J Immunol 153, 2568-2578.

Yates, R. M. \& Russell, D. G. (2005). Phagosome maturation proceeds independently of stimulation of Toll-like receptors 2 and 4 . Immunity 23, 409-417.

Edited by: G. R. Stewart 